\title{
Autofluorescent Flavoprotein Imaging of Spinal Nociceptive Activity
}

\author{
Joost L. M. Jongen, ${ }^{1,3}$ Tiziana Pederzani, ${ }^{1,3}$ Sebastiaan K. Koekkoek, ${ }^{3}$ Joël Shapiro, ${ }^{3}$ Johannes van der Burg, ${ }^{3}$ \\ Chris I. De Zeeuw, ${ }^{3,4}$ Frank J. Huygen, ${ }^{2}$ and Jan C. Holstege ${ }^{3}$ \\ Departments of ${ }^{1}$ Neurology and ${ }^{2}$ Anesthesiology, Erasmus Medical Center, 3015 CE Rotterdam, The Netherlands, ${ }^{3}$ Department of Neuroscience, Erasmus \\ Medical Center, 3015 GE Rotterdam, The Netherlands, and ${ }^{4}$ Netherlands Institute for Neuroscience, Royal Academy for Arts and Sciences, 1105 BA \\ Amsterdam, The Netherlands
}

Pain arises from activation of peripheral nociceptors, and strong noxious stimuli may cause an increase in spinal excitability called central sensitization, which is likely involved in many pathological pain states. So far, it has not been achieved to simultaneously visualize in vivo both the temporal and spatial aspects of spinal activity, including central sensitization. Using autofluorescent flavoprotein imaging (AFI), an optical technique suitable for mapping activity in nervous tissue, we demonstrate a close temporal and spatial correlation of electrically evoked nociceptive input with the spinal AFI signal, representing spinal neuronal activity. The AFI signal increases linearly with stimulation intensity. Furthermore, we found that the AFI signal was much larger in intensity and size when the same electrical stimulation was applied after the induction of central sensitization by a subcutaneous capsaicin injection. Finally, innocuous palpation of the hindpaw did not evoke an AFI response in naive animals, but after capsaicin injection a strong response was obtained. This is the first report demonstrating simultaneously the temporal and spatial propagation of spinal nociceptive activity in vivo.

\section{Introduction}

Chronic pain is an enormous clinical problem, both by the numbers of patients suffering from it and because for many chronic pain conditions there is no adequate treatment. Pain arises from activation of thinly (A $\delta$ ) or unmyelinated (C) primary afferent fibers called nociceptors, which terminate primarily in the superficial layers of the spinal cord dorsal horn. Here, the nociceptive signal is transmitted to second-order nociceptive neurons, which play an important role in organizing local reflexes and relaying the information to supraspinal centers. In 1983, using the flexor reflex in decerebrated animals, it was shown for the first time that the long-term increase in excitability after strong noxious stimuli involves changes in spinal neurons (Woolf, 1983). This phenomenon, named central sensitization, was later confirmed in human psychophysical (LaMotte et al., 1991) and animal electrophysiological studies (Ikeda et al., 2006). However, psychophysical tests are an indirect measure of spinal activity, and spinal cord electrophysiology is not capable to record the intensity and the spatial extension of the spinal signal simultaneously.

Autofluorescent flavoprotein imaging (AFI) is an optical technique suitable for monitoring metabolic activity in the superficial

Received Jan. 3, 2010; revised Feb. 1, 2010; accepted Feb. 8, 2010.

This work was supported by the Pain Knowledge Center Rotterdam/Dutch Ministry of Health (J.L.M.J.), the Dutch Organization for Medical Sciences (C.I.D.Z.), Life Sciences (C.I.D.Z.), Senter (Neuro-Bsik) (C.I.D.Z.), Prinses Beatrix Fonds (C.I.D.Z.), and the European Union (C.I.D.Z.).

J.L.M.J. and T.P. contributed equally to this work.

Correspondence should be addressed to Dr. Joost L. M. Jongen, Room Hs-609, Department of Neurology, Erasmus Medical Center, 's Gravendijkwal 230, 3015 CE Rotterdam, The Netherlands. E-mail: j.jongen@erasmusmc.nl. DOI:10.1523/JNEUROSCI.0011-10.2010

Copyright $\odot 2010$ the authors $\quad 0270-6474 / 10 / 304081-07 \$ 15.00 / 0$ areas of nervous tissue, at a high temporal and spatial resolution in vivo. Flavoproteins are mitochondrial proteins that emit green fluorescent light only in the oxidized state. In the cerebellum (Gao et al., 2006) and cerebral cortex (Shibuki et al., 2003), it has been shown, after direct electrical stimulation close to the recording site, that the light phase of the AFI signal represents postsynaptic neuronal activity. AFI clearly has advantages over other types of in vivo optical imaging. First, it does not require potentially toxic exogenous fluorescent dyes. Second, the signal intensity is high compared with nicotinamide adenine dinucleotide (NADH) imaging (Reinert et al., 2004) and intrinsic hemodynamic optical imaging (Sasaki et al., 2002). Third, AFI is a direct measure of neuronal metabolic demand and thus neuronal activity, in contrast to hemodynamic optical imaging and functional magnetic resonance imaging (fMRI) (Weber et al., 2004; Reinert et al., 2007; Tohmi et al., 2009). So far, AFI has never been applied to the spinal cord.

Since clinically relevant chronic pain disorders, like arthritis pain, cancer pain, and neuropathic pain, are all characterized both by increased pain perception within the affected area (i.e., primary hyperalgesia) as well as in the surrounding area (i.e., secondary hyperalgesia), we were looking for a way to monitor the intensity and area of spinal excitation simultaneously. We decided to use AFI to monitor spinal nociceptive activity in vivo and to record the changes in spinal excitability induced by intradermal injection of capsaicin, a nociceptor stimulant that is known to induce central sensitization (Simone et al., 1991). We found a close temporal and spatial correlation of electrically evoked nociceptive input with the spinal AFI signal, representing spinal neuronal activity. We also demonstrate, for the first time in 

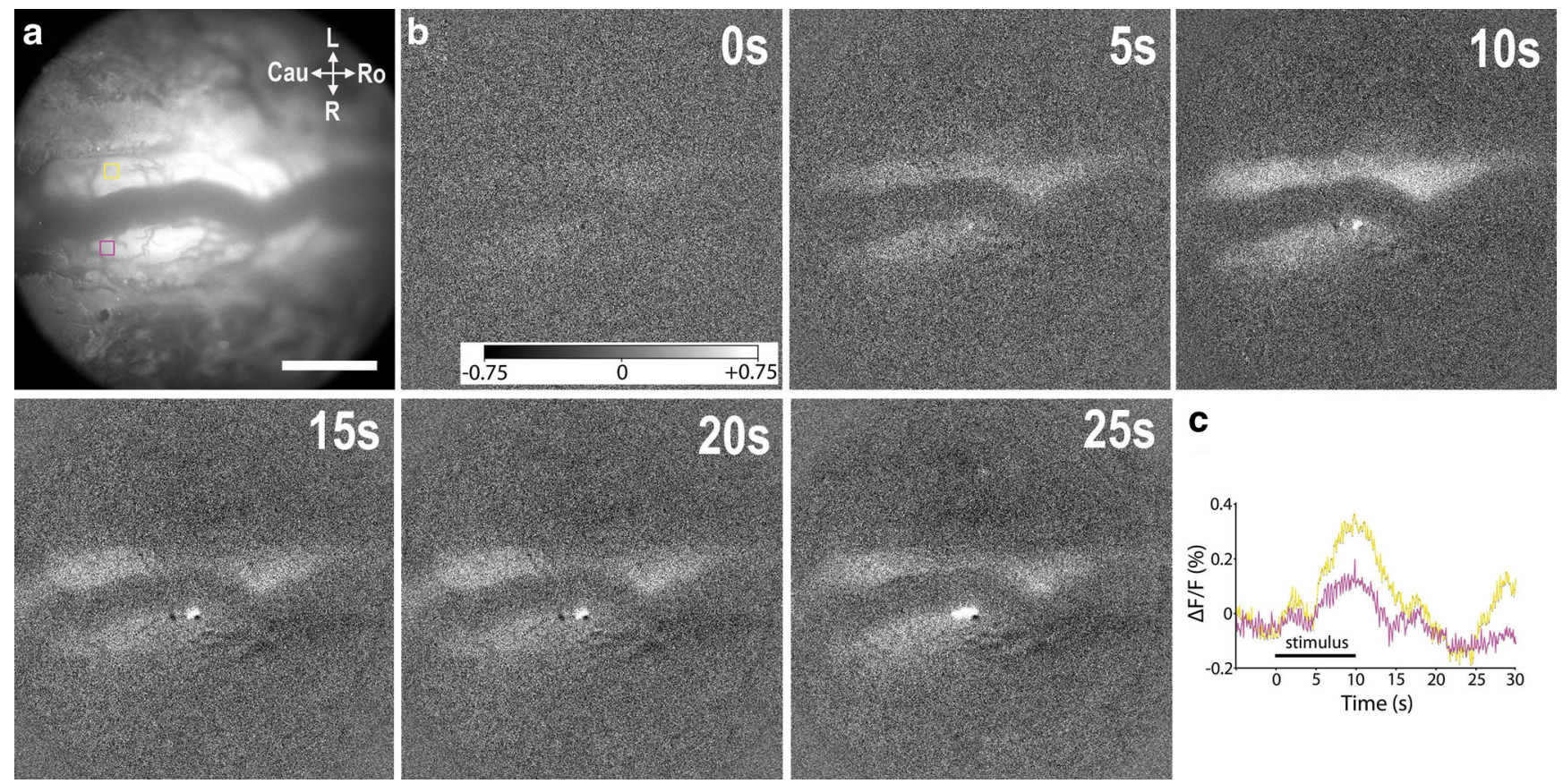

C

Figure 1. Spinal cord AFI signal after nociceptive electrical stimulation of the sciatic nerve. $\boldsymbol{a}$, Image of background fluorescence showing the dorsal surface of the spinal cord at the T13 vertebral level. The top half is left, the bottom half is right, and the dark structure in the center is a dural vein. $\boldsymbol{b}$, Subtracted $\Delta F / F$ images at various time points after start of electrical stimulation ( $4 \mathrm{~mA}$; 10 $\mathrm{Hz}$ ) of the left sciatic nerve. $\boldsymbol{c}$, Graph showing the time course of $\Delta F / F$ in the yellow [left (i.e., ipsilateral or stimulated side)] and purple [right (i.e., contralateral)] square selections in $\boldsymbol{a}$. Scale bar, 1 $\mathrm{mm}$. Grayscale bar ranging from $-0.75 \%$ (black) to $+0.75 \%$ (white) of the 16 bit range. Cau, Caudal; Ro, rostral; L, left; R, right.

vivo, simultaneously an increased intensity and area of spinal activity attributable to central sensitization.

\section{Materials and Methods}

Animal preparation. All animal experimentation was approved by and conducted in conformity with the Animal Ethics Committee of Erasmus Medical Center Rotterdam. To obtain AFI responses, young adult rats (Harlan) were anesthetized with an intraperitoneal injection of $2 \mathrm{mg} / \mathrm{g}$ urethane (Sigma-Aldrich). A tracheotomy was performed, and a 1.6$\mathrm{mm}$-wide tracheal cannula was inserted for mechanical ventilation during the recordings. Then, a $1-\mathrm{cm}$-wide silicon cuff containing a bipolar electrode was placed around the proximal left sciatic nerve. A laminectomy was performed at the 13th thoracic vertebra and the lower lumbar segments of the spinal cord were visualized through the intact dura mater. The vertebral column was fixed in a spinal frame. Then the animals were paralyzed with an intramuscular injection of $4 \mu \mathrm{g} / \mathrm{g}$ pancuronium bromide (Organon). A bilateral pneumothorax was induced with 0.9 $\mathrm{mm}$ needles, and rats were mechanically ventilated at a rate of 60 strokes/ min. Colorectal temperature was kept at $\sim 37.5^{\circ} \mathrm{C}$ with a feedback controlled heating blanket. The heart rate was continuously monitored by three subcutaneous electrocardiogram electrodes.

The sciatic nerve was stimulated with $10 \mathrm{~s}$ trains of $10 \mathrm{~Hz}$ electrical stimuli, at a strength of $1,2.5$, or $4 \mathrm{~mA}$. A recording always consisted of $10 \mathrm{~s}$ of baseline, $10 \mathrm{~s}$ of recording during electrical stimulation, and $20 \mathrm{~s}$ of recording after electrical stimulation. During the $40 \mathrm{~s}$ lasting recordings, mechanical ventilation was temporarily switched off, to reduce spinal movement artifacts. Between recordings, animals were allowed to recover for 5 min until the next recording.

For the first capsaicin experiment, rats were injected in the left footpad with either $25 \mu \mathrm{g}$ of capsaicin (Sigma-Aldrich) or solvent (vehicle), consisting of $100 \mu \mathrm{l}$ of pure ethanol mixed with $900 \mu \mathrm{l}$ of Tween 80. Spinal AFI responses to $10 \mathrm{~s}$ trains of $2.5 \mathrm{~mA}, 10 \mathrm{~Hz}$ stimulation of the sciatic nerve were recorded every $5 \mathrm{~min}$ for up to $1 \mathrm{~h}$ after injection. In another group of rats, we compared AFI responses to $10 \mathrm{~s}, 1 \mathrm{~Hz}$ innocuous palpation of the left hindpaw, before capsaicin injection and $32 \mathrm{~min}$ after capsaicin injection. Innocuous palpation consisted of gentle touches manually applied to the plantar surface of the hindpaw.

Optical imaging. The flavoprotein signal was captured with a sensitive, high-speed electron-multiplying charge-coupled device camera (Roper Scientific). The camera was mounted on an upright microscope with a $5 \times / 0.12$ objective (Carl Zeiss). The lens was focused on the lateral braches of the large central dural vein, and then the focal plane was adjusted $150 \mu \mathrm{m}$ downward, which depth corresponds with the superficial dorsal horn (Sasaki et al., 2002). A mercury lamp (Carl Zeiss) served as a light source and a $445 \pm 35 \mathrm{~nm}$ excitation filter, a $>500 \mathrm{~nm}$ dichroic beam splitter, and a $>515 \mathrm{~nm}$ emission filter were used (supplemental Fig. 1, available at www. jneurosci.org as supplemental material).

Data analysis. After image acquisition, the first 50 frames were discarded from the analysis. Then a series of difference images was created by subtracting the average of the 50 frames preceding electrical stimulation (control frames) from each control and experimental frame. These difference images were then divided by the control average, resulting in images in which the intensity value of each pixel reflects the $\Delta F / F$ change in fluorescence intensity relative to the average of the control frames (Reinert et al., 2004). To quantify the time course and intensity of the autofluorescence response, regions of interest (ROIs) of $20 \times 20$ pixels were selected on the left (ipsilateral or stimulated) side and right (contralateral) side of the spinal cord. ROIs in the bottom left corner of the frame were subtracted from the primary ROIs to correct for fluctuations in background fluorescence or light intensity.

For the capsaicin experiment, first the $\Delta F / F$ of the responses was determined. Then, the areas of excitation at $32 \mathrm{~min}$ after injection were compared between capsaicin- and vehicle-treated animals. To determine these areas, all subtracted $\Delta F / F$ images were binarized using the same threshold. Borders were drawn by a researcher, who was blind to the treatment.

Statistical analysis was performed using GraphPad Prism, version 5.0a, software. Figures were composed in Adobe Photoshop CS3, version 10.0, software. Adjustments were made only to brightness and contrast and applied evenly to all panels of a figure. 

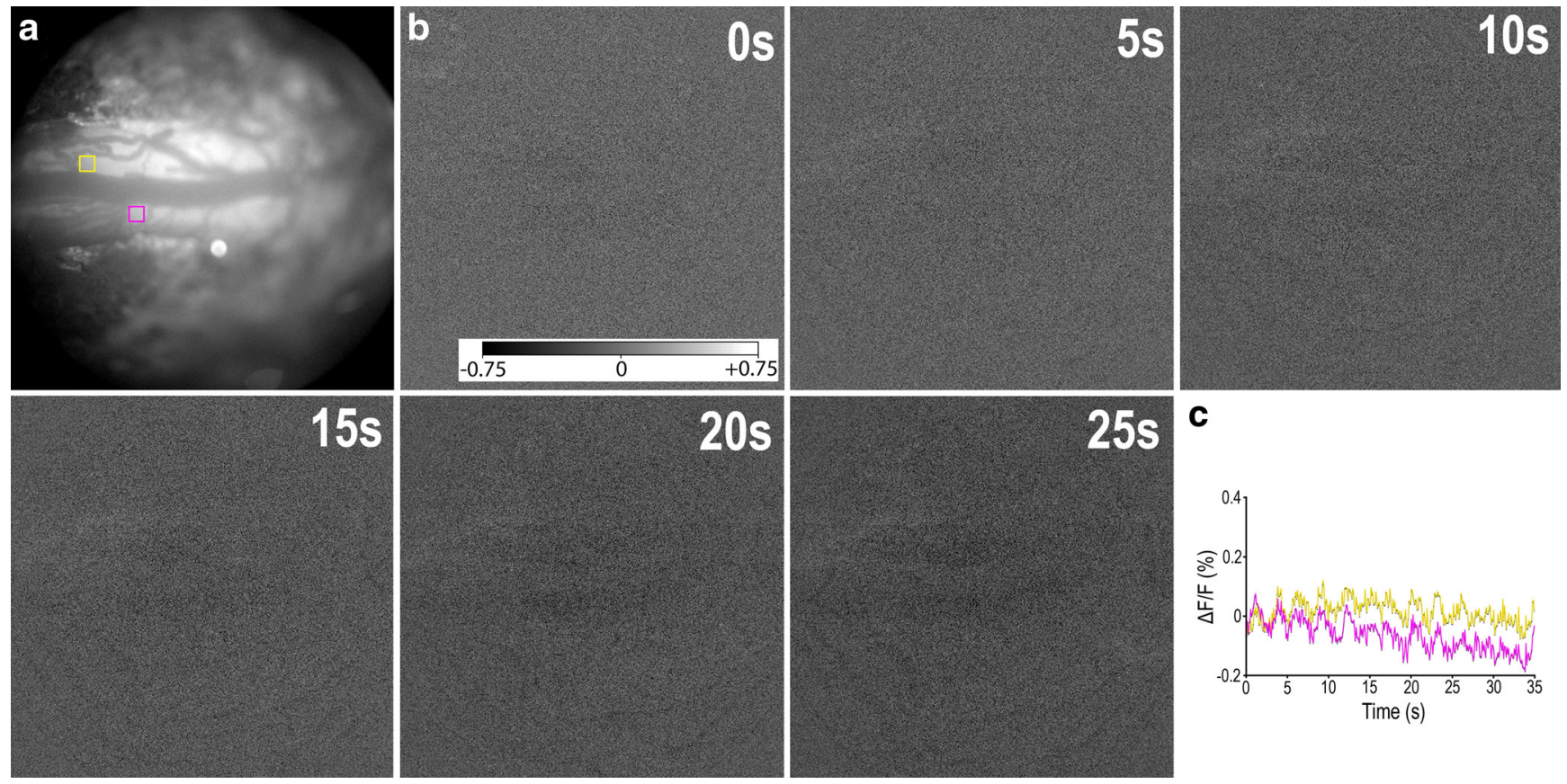

Figure 2. Spinal cord AFI signal in rats with a sciatic nerve electrode cuff but without electrical stimulation. $\boldsymbol{a}$, Image of background fluorescence showing the dorsal surface of the spinal cord at the T13 vertebral level. The top half is left, the bottom half is right, and the dark structure in the center is a dural vein. $\boldsymbol{b}$, Subtracted $\Delta F / F$ images at various time points after start of the recording. c, Graph showing the time course of $\Delta F / F$ in the yellow [left (i.e., ipsilateral or stimulated side)] and purple [right (i.e., contralateral)] square selections in $\boldsymbol{a}$. Scale bar, $1 \mathrm{~mm}$. Grayscale bar ranging from $-0.75 \%$ (black) to $+0.75 \%$ (white) of the 16 bit range.

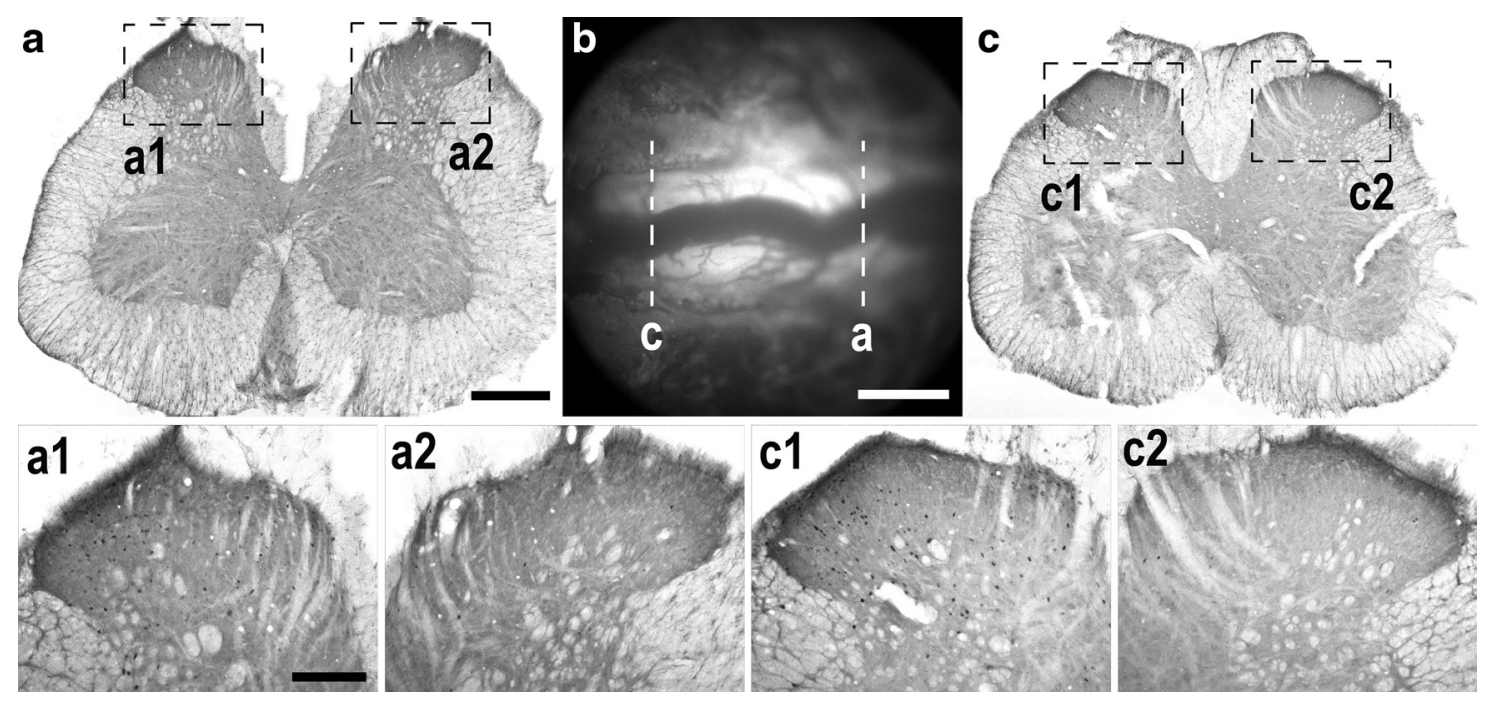

Figure 3. Correlation of spinal AFI signal with c-Fos expression. $\boldsymbol{a}, \mathrm{L} 4$ section, the approximate position of which is indicated in $\boldsymbol{b}$, processed for c-Fos immunohistochemistry. $\boldsymbol{c}$, Similarly, a $\mathrm{L} 6$ section is shown. $\boldsymbol{a} 1$ and $\boldsymbol{a} \mathbf{2}$, and $\mathbf{c} \mathbf{1}$ and $\boldsymbol{c} \mathbf{2}$ are enlargements of the dorsal horn in $\boldsymbol{a}$ and $\boldsymbol{c}$, respectively. Scale bars: $\boldsymbol{a}, 500 \mu \mathrm{m} ; \boldsymbol{b}, 1 \mathrm{~mm} ; \boldsymbol{a} \mathbf{1}, 200 \mu \mathrm{m}$.

Immunohistochemistry. Directly after flavoprotein imaging, two rats were transcardially perfused with $250 \mathrm{ml}$ of phosphate buffer $(\mathrm{PB})(0.025 \mathrm{M})$, pH 7.3, containing $0.8 \% \mathrm{NaCl}, 0.8 \%$ sucrose, and $0.4 \%$ D-glucose, followed by $400 \mathrm{ml}$ of PB $(0.05 \mathrm{M})$ containing $4 \%$ paraformaldehyde and $400 \mathrm{ml}$ of the same fixative also containing $15 \%$ sucrose, both at $4^{\circ} \mathrm{C}$. After perfusion, the lumbar spinal cord was removed and postfixed at $4^{\circ} \mathrm{C}$ for $2 \mathrm{~h}$ in the fixative containing $15 \%$ sucrose, transferred to $30 \%$ sucrose in $0.05 \mathrm{M} \mathrm{PB}$, and left overnight at $4^{\circ} \mathrm{C}$. Sections were cut at $40 \mu \mathrm{m}$ on a freezing microtome, rinsed in PBS, and processed for immunohistochemistry. They were preincubated $\left(1 \mathrm{~h}\right.$ at $\left.20^{\circ} \mathrm{C}\right)$ with $10 \%$ normal serum (NS) and $0.3 \%$ Triton in PBS, followed by incubation $(60 \mathrm{~h}$ at $4^{\circ} \mathrm{C}$ ) in $2 \% \mathrm{NS}, 0.4 \%$ Triton, and PBS with rabbit anti-c-Fos (1: 10,000; PC 38; Calbiochem). Subsequently, sections were rinsed in PBS and incubated $\left(1.5 \mathrm{~h}\right.$ at $\left.20^{\circ} \mathrm{C}\right)$ with biotinylated goat anti-rabbit
(1:200; Vector Laboratories), 2\% NS and 0.4\% Triton in PBS. Finally, they were rinsed in $\mathrm{PBS}$ and placed in $\mathrm{ABC}$ reagent (avidin-biotin peroxidase; Vector Laboratories) containing $0.4 \%$ Triton $(1.5 \mathrm{~h}$ at $20^{\circ} \mathrm{C}$ ), rinsed again with $\mathrm{PBS}$ and then with $0.05 \mathrm{M} \mathrm{PB}$, and reacted with 3',3-diaminobenzidine tetrahydrochloride and $0.005 \% \mathrm{H}_{2} \mathrm{O}_{2}$ dissolved in PB. Subsequently, sections were rinsed in $\mathrm{PB}$ and mounted on a slide and coverslipped, using Permount (Thermo Fisher Scientific) as mounting medium.

\section{Results}

A typical recording of a $10 \mathrm{~s}, 4 \mathrm{~mA}, 10 \mathrm{~Hz}$ electrical stimulation of the sciatic nerve showed a steep increase in spinal cord fluorescence (light phase) immediately after the start of the electrical 
stimulation, followed by a decrease below baseline (dark phase), that returned to baseline at the end of the recording (Fig. 1; supplemental Videos 1, 2, available at www.jneurosci.org as supplemental material). This stimulus intensity is approximately three times C-fiber threshold, which has been shown to approximate 1 $\mathrm{mA}$ in rats in vivo (Rygh et al., 2000; Liu et al., 2007), thus exciting the majority of $\mathrm{A} \delta$ - and C-fibers. The pattern of activity that we observed is similar to AFI responses elsewhere in the nervous system (Murakami et al., 2004; Reinert et al., 2004; Gao et al., 2006). The change in fluorescence was much more pronounced on the stimulated side than on the contralateral side.

We then conducted a series of control experiments. As a first control, recordings were made without electrical stimulation in six animals. Thus, it was shown that the absence of ventilation during the recordings hardly affected the AFI signal (Fig. 2). As a second control, filter settings outside the range of excitation and emission wavelengths for flavoproteins were used. In this case, no AFI signal was detected. As a final control, the sciatic nerve was blocked by local application of $0.4 \mathrm{ml}$ of $20 \mathrm{mg} / \mathrm{ml}$ lidocaine. In this situation, a standard nociceptive electrical stimulation did not show any change during the entire course of the recording $(n=2)$ (data not shown), indicating that our stimulation was indeed selective. These experiments thus confirmed the close temporal correlation of the AFI signal with spinal activity induced by nociceptive electrical stimulation of the sciatic nerve.

To verify whether the distribution of the AFI signal corresponded with c-Fos, a chemical marker of spinal nociceptive activity (Hunt et al., 1987), two rats were transcardially perfused after AFI recordings and processed for c-Fos immunohistochemistry. Both the AFI signal and the increase in c-Fos-labeled neurons were mainly localized in the ipsilateral spinal segments L4-L6, which is the projection area of sciatic nerve afferents (Fig. 3). A much smaller increase in c-Fos was observed on the contralateral side (Fig. $3 a$ ). This experiment thus confirmed the close spatial correlation of the AFI signal with spinal activity induced by nociceptive electrical stimulation of the sciatic nerve.

The AFI signal was highly dependent on stimulation intensity. Increasing the stimulation intensity from 1 to $4 \mathrm{~mA}$, causing activation of increasing numbers of $\mathrm{A} \delta$ - and C-fibers, resulted in an almost threefold linear increase in the intensity of the light phase of the AFI signal on the stimulated side, whereas the AFI signal on the contralateral side did not increase with increasing
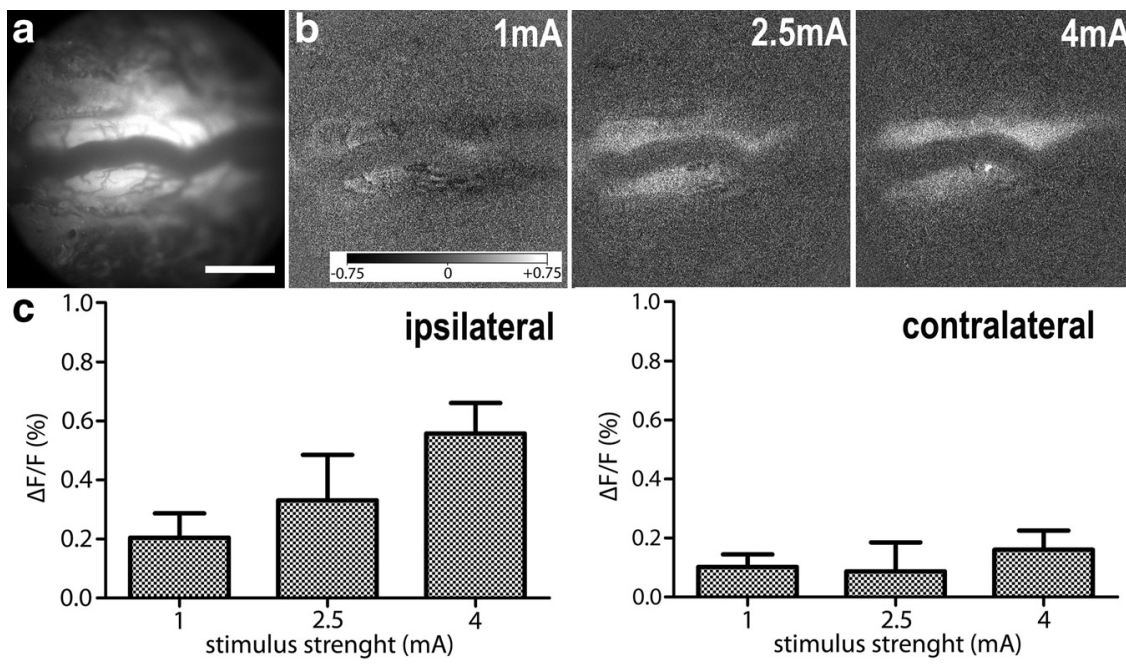

Figure 4. Dependency of spinal AFI signal on stimulation intensity. $\boldsymbol{a}$, Image of background fluorescence of the dorsal surface of the spinal cord at T13. $\boldsymbol{b}$, Subtracted $\Delta F / F$ images $10 \mathrm{~s}$ after start of electrical stimulation, using various stimulation intensities. c, Mean $\Delta F / F$ of the light phase (frame 180-200) in square selections on the ipsilateral and contralateral side at the L4-L6 spinal level. $\Delta F / F$ values were created from the averages of two to four recordings in each of nine animals. Scale bar, $1 \mathrm{~mm}$. Grayscale bar ranging from $-0.75 \%$ (black) to $+0.75 \%$ (white) of the 16 bit range. Error bars indicate SEM.
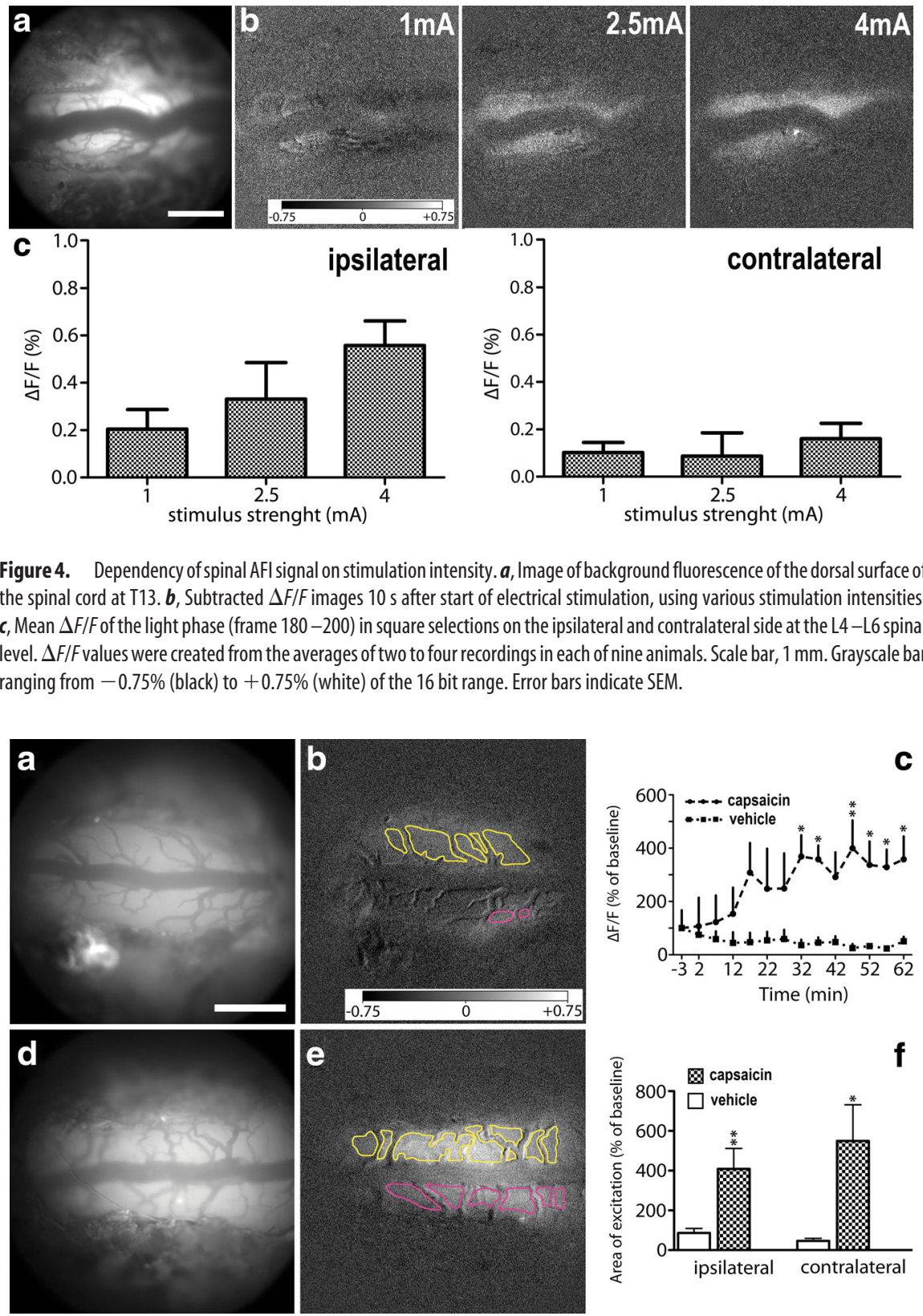

Figure 5. Intensity of the AFI signal and area of excitation, after sciatic nerve electrical stimulation in rats with intraplantar vehicle or capsaicin injection. $\boldsymbol{a}, \boldsymbol{d}$, Images of background fluorescence of the dorsal surface of the spinal cord at T13 of a vehicle- $(\boldsymbol{a})$ and capsaicin-injected rat (d), at 32 min after injection. $\boldsymbol{b}, \boldsymbol{e}$, Areas of excitation on the ipsilateral (yellow) and contralateral (purple) side, at 32 min after injection. c, Time course of the intensity of the AFI signal after injection, as a percentage of $\Delta F / F$ before injection (baseline), in square selections on the ipsilateral side at the $L 4-L 6$ spinal level. $f$, Mean areas of excitation at $32 \mathrm{~min}$ after injection, as a percentage of the areas before injection (baseline). Scale bar, $1 \mathrm{~mm}$. Grayscale bar ranging from $-0.75 \%$ (black) to $+0.75 \%$ (white) of the 16 bit range. Error bars indicate SEM. ${ }^{*} p<0.05$, ${ }^{* *} p<0.01$, using Bonferroni's correction only in $c$.

stimulation intensity (Fig. 4). A linear regression to the stimulus intensity yielded a significant fit $\left(n=9 ; R^{2}=0.16, F\right.$ test, $p=$ 0.04 ; slope $=0.12 \% \Delta F / F \cdot \mathrm{mA}^{-1}$ ) on the stimulated side. When the stimulation intensity was increased to $8 \mathrm{~mA}$, no additional increase in AFI signal was observed compared with $4 \mathrm{~mA}$ (data not shown). Furthermore, innocuous palpation causing mainly $\mathrm{A} \beta$-fiber activation did not cause an AFI response (see below).

After having validated the technique, we focused on visualizing the effects of central sensitization. We investigated whether the AFI technique is able to detect the increased excitability of the 

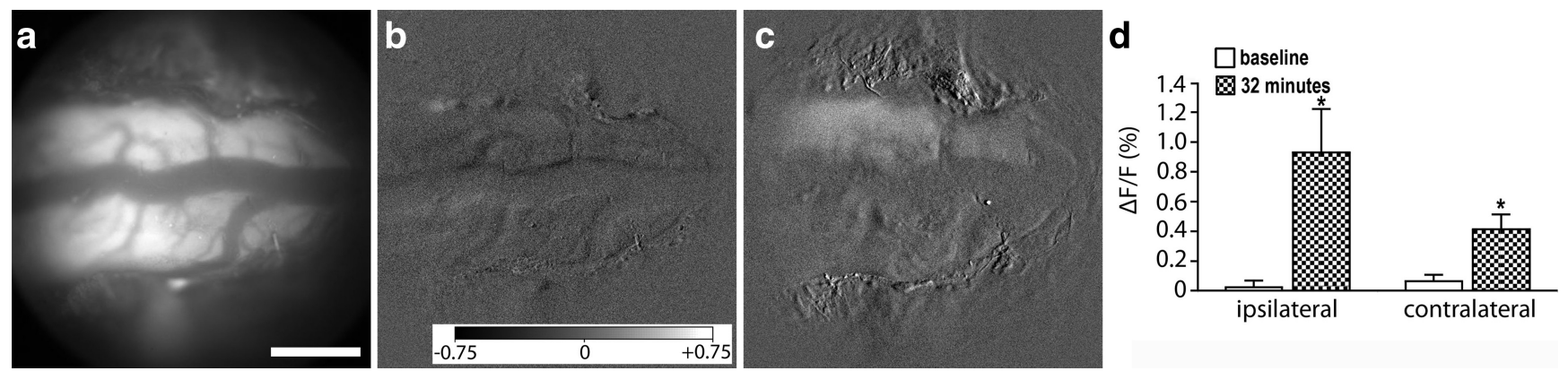

Figure 6. Intensity of the AFI signal after innocuous palpation in rats at baseline and at 32 min after capsaicin injection. $\boldsymbol{a}$, Image of background fluorescence of the dorsal surface of the spinal cord at T13. $\boldsymbol{b}, \boldsymbol{c}$, Subtracted $\Delta$ F/Fimages $10 \mathrm{~s}$ after start of innocuous palpation, at baseline (b) and at 32 min after injection of capsaicin (c). $\boldsymbol{d}$, Mean $\Delta F / F$ of the light phase (frame $180-200)$ in square selections on the ipsilateral and contralateral side at the L4-L6 spinal level, at baseline and at $32 \mathrm{~min}$ after capsaicin injection. Scale bar, $1 \mathrm{~mm}$. Grayscale bar ranging from $-0.75 \%$ (black) to $+0.75 \%$ (white) of the 16 bit range. Error bars indicate SEM. * $p<0.05$.

spinal cord (i.e., central sensitization) after intradermal injection of capsaicin, the pungent ingredient of chili peppers. For this purpose, one group of animals received intradermal capsaicin and another group intradermal injection of vehicle solution in the left hindpaw. AFI responses to $10 \mathrm{~s}, 2.5 \mathrm{~mA}, 10 \mathrm{~Hz}$ sciatic nerve electrical stimuli were recorded every $5 \mathrm{~min}$ for up to $1 \mathrm{~h}$. We thus demonstrated a slowly developing but robust (approximately four times) increase in AFI intensity after capsaicin injection but not after vehicle injection (Fig. $5 a-c$ ). A repeated-measures ANOVA showed that the overall difference between the two treatments was statistically significant $(n=8+8 ; p=0.02)$. Using post hoc analysis, it was determined that statistical significance was reached $32 \mathrm{~min}$ after injection. Second, we demonstrated an equally robust (four to six times) increased area of excitation $32 \mathrm{~min}$ after injection, induced by the capsaicin injection (Fig. $5 d-f$ ). Unpaired $t$ tests yielded significant differences between the areas of excitation of vehicle and capsaicin, on both sides of the spinal cord.

Since it is known that after capsaicin injection also innocuous stimuli may elicit nociceptive activity in the spinal superficial dorsal horn (Koltzenburg et al., 1992; Mantyh et al., 1997; Latremoliere and Woolf, 2009), we investigated AFI responses to $10 \mathrm{~s}, 1 \mathrm{~Hz}$ innocuous palpation of the left hindpaw, at baseline and $32 \mathrm{~min}$ after capsaicin injection. AFI responses to innocuous palpation before capsaicin injection were not different from recordings without stimulation on both the ipsilateral and contralateral side $(n=5 ; p>0.05)$. However, there was a robust AFI response to palpation $32 \mathrm{~min}$ after capsaicin injection, which was most pronounced on the ipsilateral side (Fig. 6; supplemental Videos 3, 4, available at www.jneurosci.org as supplemental material). A paired $t$ test yielded a significant difference between AFI responses to palpation at baseline and at 32 min after capsaicin injection on both the ipsilateral and contralateral side $(n=5 ; p<0.05)$.

\section{Discussion}

This is the first report using AFI to study spinal cord nociceptive activity in vivo. We demonstrate a close temporal and spatial correlation of lumbar spinal cord AFI responses to electrically evoked input from the sciatic nerve, representing spinal neuronal activity. The AFI signal increased linearly with increasing stimulus intensity. In addition, AFI enabled us to simultaneously visualize a capsaicin-induced increase in intensity and area of excitation of spinal cord responses to electrically evoked nociceptive input. Finally, using AFI, we demonstrated a robust response to innocuous palpation after capsaicin injection, but not in naive animals.

Electrical stimulus intensities ranging from one to more than three times C-fiber threshold were applied in our study (Rygh et al., 2000; Liu et al., 2007). Additionally, we used innocuous palpation causing mainly $\mathrm{A} \beta$-fiber stimulation in naive animals. Since only the former stimuli evoked AFI responses in naive animals, we conclude that the change in AFI activity in the spinal cord that we observed in naive animals mainly results from A $\delta$ and C-fiber activation. The responses that we observed after capsaicin injection may result from activation of all types of sensory fibers [i.e., $\mathrm{A} \delta, \mathrm{C}, \mathrm{A} \beta$, and $\mathrm{C}$ low-threshold mechanoreceptors (Seal et al., 2009)].

Spinal cord AFI is more challenging than AFI of the brain because of movement artifacts caused by the nearby heart and respiration. However, using a muscle relaxant, mechanical ventilation, and a pneumothorax, this problem can be overcome. The close temporal correlation of the spinal AFI response to electrical input is in agreement with other in vivo AFI studies using direct electrical stimulation of the somatosensory cortex (Shibuki et al., 2003) or cerebellum (Reinert et al., 2004; Gao et al., 2006; Wang et al., 2009). The somatotopic distribution of the AFI response that we found after sciatic nerve stimulation is in agreement with in vivo AFI studies using tactile, auditory, and visual stimuli and AFI recordings from the somatosensory cortex (Shibuki et al., 2003; Murakami et al., 2004), barrel cortex (Weber et al., 2004), auditory cortex (Takahashi et al., 2006; Kubota et al., 2008), or visual cortex (Tohmi et al., 2006; Husson et al., 2007), respectively. Furthermore, the absence of an AFI response after electrical blockade of the sciatic nerve by lidocaine indicated that the AFI signal was indeed generated by sciatic nerve input. Finally, the close spatial correlation of the spinal AFI signal with c-Fos expression confirmed that the spinal AFI signal is representative of spinal nociceptive activity, since c-Fos is expressed after nociceptive stimulation but not after non-nociceptive stimulation (Hunt et al., 1987). Contralateral AFI activity, although low compared with ipsilateral AFI activity, matched contralateral c-Fos expression, which has been described after acute and chronic stimulation of the orofacial region (Zhou et al., 1999; Ono et al., 2009) and the hindlimb (Hudspith et al., 1999; Ro et al., 2004). Contralateral activity is likely attributable to polysynaptic mechanisms.

In our study, we only used the early or light phase of the AFI response, which is considered to result from postsynaptic neuronal excitation (Reinert et al., 2007). We adjusted the focal plane to 
the most superficial areas of the rat dorsal spinal cord [i.e., lamina I and II according to Molander et al. (1984)]. Since AFI is an optical method and optical responses decrease with increased depth of the focal plane (Sasaki et al., 2002), we hypothesize that the largest contribution of the spinal cord AFI response is generated by projection neurons and local interneurons in these superficial laminae (Jongen et al., 2007; Reinert et al., 2007). However, we cannot exclude that also nerve terminals in the superficial dorsal horn or neurons and nerve terminals in the deeper laminae contribute to the spinal cord AFI response.

The linear increase of the AFI response with increasing stimulus intensities (at least up to $4 \mathrm{~mA}, 10 \mathrm{~Hz}$ stimulus intensities) enabled us to quantify the effects of intradermal capsaicin injection on spinal excitability. Since repetitive electrical stimulation at C-fiber strength may in itself give rise to sensitization (Ikeda et al., 2006), we used vehicle-injected animals as controls, in which AFI responses remained unchanged. LaMotte et al. (1991) have previously described an increased area of punctate hyperalgesia immediately after capsaicin injection, which grew to a maximum within $30 \mathrm{~min}$. Ikeda et al. (2006) described a gradually developing long-term potentiation of $\mathrm{C}$-fiber evoked field potentials in the superficial dorsal horn in vivo, which reached a maximum $1 \mathrm{~h}$ after capsaicin injection. The time course of the increases in electrical and behavioral responses that these authors found is thus comparable with the time course of the AFI response that we describe here. The magnitude of the increased responses using AFI is even greater than using electrophysiology, suggesting that AFI may be a more sensitive technique. However, central sensitization, which is defined as "an enhanced responsiveness of nociceptive neurons in the CNS to their normal afferent input" (Sandkühler, 2007; Latremoliere and Woolf, 2009), is not only characterized by an increased amplitude of a response but also by an increased receptive field. This was demonstrated for the first time in 1983, when a prolonged and strong nociceptive stimulus to one hindpaw caused a hyperresponsive flexor reflex not only on the ipsilateral but also on the contralateral side (Woolf, 1983). Exploiting the features of AFI, we now demonstrate for the first time both dimensions of spinal dorsal horn plasticity in a single recording. Thus, in vivo AFI of the spinal cord has an advantage over one-dimensional spinal cord electrophysiology as well as over animal behavioral studies, which are notoriously liable to external factors. We therefore suggest that AFI may contribute significantly to a better understanding of the mechanisms underlying chronic pain, more specifically spinal sensitization.

Currently, both the temporal and spatial resolution of AFI are many times higher than that of fMRI, rendering the latter incapable of detailed studies of spinal nociceptive activity in small animals. Therefore AFI is an excellent technique to detect changes in spinal excitability (i.e., central sensitization) in rat or mouse models of clinically relevant pain disorders. Central sensitization of normal afferent input is hypothesized to contribute to chronic pain disorders like arthritis pain, cancer pain, and neuropathic pain (Sandkühler, 2007). Hence, our methodology may be applied to screen the efficacy of analgesic compounds or interventions to reduce central sensitization in animal models of the aforementioned chronic pain conditions. Ultimately, using fiber optics, AFI might also be applied in humans as a minimally invasive procedure. This would allow for a new and objective assessment of the effect of various therapeutic intervention techniques, which are notoriously difficult to evaluate objectively.

\section{References}

Gao W, Chen G, Reinert KC, Ebner TJ (2006) Cerebellar cortical molecular layer inhibition is organized in parasagittal zones. J Neurosci 26:83778387.

Hudspith MJ, Harrisson S, Smith G, Bountra C, Elliot PJ, Birch PJ, Hunt SP, Munglani R (1999) Effect of post-injury NMDA antagonist treatment on long-term Fos expression and hyperalgesia in a model of chronic neuropathic pain. Brain Res 822:220-227.

Hunt SP, Pini A, Evan G (1987) Induction of c-fos-like protein in spinal cord neurons following sensory stimulation. Nature 328:632-634.

Husson TR, Mallik AK, Zhang JX, Issa NP (2007) Functional imaging of primary visual cortex using flavoprotein autofluorescence. J Neurosci 27:8665-8675.

Ikeda H, Stark J, Fischer H, Wagner M, Drdla R, Jäger T, Sandkühler J (2006) Synaptic amplifier of inflammatory pain in the spinal dorsal horn. Science 312:1659-1662.

Jongen JL, Jaarsma D, Hossaini M, Natarajan D, Haasdijk ED, Holstege JC (2007) Distribution of RET immunoreactivity in the rodent spinal cord and changes after nerve injury. J Comp Neurol 500:1136-1153.

Koltzenburg M, Lundberg LE, Torebjörk HE (1992) Dynamic and static components of mechanical hyperalgesia in human hairy skin. Pain 51:207-219.

Kubota Y, Kamatani D, Tsukano H, Ohshima S, Takahashi K, Hishida R, Kudoh M, Takahashi S, Shibuki K (2008) Transcranial photo-inactivation of neural activities in the mouse auditory cortex. Neurosci Res 60: 422-430.

LaMotte RH, Shain CN, Simone DA, Tsai EF (1991) Neurogenic hyperalgesia: psychophysical studies of underlying mechanisms. J Neurophysiol 66: $190-211$.

Latremoliere A, Woolf CJ (2009) Central sensitization: a generator of pain hypersensitivity by central neural plasticity. J Pain 10:895-926.

Liu FY, Xing GG, Qu XX, Xu IS, Han JS, Wan Y (2007) Roles of 5-hydroxytryptamine (5-HT) receptor subtypes in the inhibitory effects of 5-HT on C-fiber responses of spinal wide dynamic range neurons in rats. J Pharmacol Exp Ther 321:1046-1053.

Mantyh PW, Rogers SD, Honore P, Allen BJ, Ghilardi JR, Li J, Daughters RS, Lappi DA, Wiley RG, Simone DA (1997) Inhibition of hyperalgesia by ablation of lamina I spinal neurons expressing the substance P receptor. Science 278:275-279.

Molander C, Xu Q, Grant G (1984) The cytoarchitectonic organization of the spinal cord in the rat. I. The lower thoracic and lumbosacral cord. J Comp Neurol 230:133-141.

Murakami H, Kamatani D, Hishida R, Takao T, Kudoh M, Kawaguchi T, Tanaka R, Shibuki K (2004) Short-term plasticity visualized with flavoprotein autofluorescence in the somatosensory cortex of anaesthetized rats. Eur J Neurosci 19:1352-1360.

Ono K, Harano N, Nagahata S, Seta Y, Tsujisawa T, Inenaga K, Nakanishi O (2009) Behavioral characteristics and c-Fos expression in the medullary dorsal horn in a rat model for orofacial cancer pain. Eur J Pain 13:373-379.

Reinert KC, Dunbar RL, Gao W, Chen G, Ebner TJ (2004) Flavoprotein autofluorescence imaging of neuronal activation in the cerebellar cortex in vivo. J Neurophysiol 92:199-211.

Reinert KC, Gao W, Chen G, Ebner TJ (2007) Flavoprotein autofluorescence imaging in the cerebellar cortex in vivo. J Neurosci Res 85:3221-3232.

Ro LS, Li HY, Huang KF, Chen ST (2004) Territorial and extra-territorial distribution of Fos protein in the lumbar spinal dorsal horn neurons in rats with chronic constriction nerve injuries. Brain Res 1004:177-187.

Rygh LJ, Kontinen VK, Suzuki R, Dickenson AH (2000) Different increase in C-fibre evoked responses after nociceptive conditioning stimulation in sham-operated and neuropathic rats. Neurosci Lett 288:99-102.

Sandkühler J (2007) Understanding LTP in pain pathways. Mol Pain 3:9.

Sasaki S, Yazawa I, Miyakawa N, Mochida H, Shinomiya K, Kamino K, Momose-Sato Y, Sato K (2002) Optical imaging of intrinsic signals induced by peripheral nerve stimulation in the in vivo rat spinal cord. Neuroimage 17:1240-1255.

Seal RP, Wang X, Guan Y, Raja SN, Woodbury CJ, Basbaum AI, Edwards RH (2009) Injury-induced mechanical hypersensitivity requires C-low threshold mechanoreceptors. Nature 462:651-655.

Shibuki K, Hishida R, Murakami H, Kudoh M, Kawaguchi T, Watanabe M, 
Watanabe S, Kouuchi T, Tanaka R (2003) Dynamic imaging of somatosensory cortical activity in the rat visualized by flavoprotein autofluorescence. J Physiol 549:919-927.

Simone DA, Sorkin LS, Oh U, Chung JM, Owens C, LaMotte RH, Willis WD (1991) Neurogenic hyperalgesia: central neural correlates in responses of spinothalamic tract neurons. J Neurophysiol 66:228-246.

Takahashi K, Hishida R, Kubota Y, Kudoh M, Takahashi S, Shibuki K (2006) Transcranial fluorescence imaging of auditory cortical plasticity regulated by acoustic environments in mice. Eur J Neurosci 23:1365-1376.

Tohmi M, Kitaura H, Komagata S, Kudoh M, Shibuki K (2006) Enduring critical period plasticity visualized by transcranial flavoprotein imaging in mouse primary visual cortex. J Neurosci 26:11775-11785.

Tohmi M, Takahashi K, Kubota Y, Hishida R, Shibuki K (2009) Transcra- nial flavoprotein fluorescence imaging of mouse cortical activity and plasticity. J Neurochem 109 [Suppl 1]:S3-S9.

Wang X, Chen G, Gao W, Ebner T (2009) Long-term potentiation of the responses to parallel fiber stimulation in mouse cerebellar cortex in vivo. Neuroscience 162:713-722.

Weber B, Burger C, Wyss MT, von Schulthess GK, Scheffold F, Buck A (2004) Optical imaging of the spatiotemporal dynamics of cerebral blood flow and oxidative metabolism in the rat barrel cortex. Eur J Neurosci 20:2664-2670.

Woolf CJ (1983) Evidence for a central component of post-injury pain hypersensitivity. Nature 306:686-688.

Zhou Q, Imbe H, Dubner R, Ren K (1999) Persistent Fos protein expression after orofacial deep or cutaneous tissue inflammation in rats: implications for persistent orofacial pain. J Comp Neurol 412:276-291. 